\title{
Article
}

\section{Risk Factors for Pulmonary Embolism in Patients with Paralysis and Deep Venous Thrombosis}

\author{
Karsten Keller ${ }^{1,2,3, * \mathbb{D}}$, Jens Wöllner ${ }^{4,5}$, Volker H. Schmitt ${ }^{1,6} \mathbb{D}^{\mathbb{D}}$, Mir A. Ostad ${ }^{1}$, Ingo Sagoschen ${ }^{1}$, \\ Thomas Münzel ${ }^{1,6}\left(\mathbb{0}\right.$, Christine Espinola-Klein ${ }^{1,2}$ and Lukas Hobohm ${ }^{1,2}$ \\ 1 Department of Cardiology, Cardiology I, University Medical Center Mainz \\ (Johannes Gutenberg-University Mainz), 55131 Mainz, Germany; \\ Volker.Schmitt@unimedizin-mainz.de (V.H.S.); Ostad@uni-mainz.de (M.A.O.); \\ Ingo.Sagoschen@unimedizin-mainz.de (I.S.); tmuenzel@uni-mainz.de (T.M.); \\ espinola@uni-mainz.de (C.E.-K.); lukas.hobohm@unimedizin-mainz.de (L.H.) \\ 2 Center for Thrombosis and Hemostasis (CTH), University Medical Center Mainz \\ (Johannes Gutenberg-University Mainz), 55131 Mainz, Germany \\ 3 Medical Clinic VII, Department of Sports Medicine, University Hospital Heidelberg, \\ 69120 Heidelberg, Germany \\ 4 Swiss Paraplegic Center Nottwil, Department of Neuro-Urology, 6207 Nottwil, Switzerland; \\ jens.woellner@paraplegie.ch \\ 5 Department of Urology and Pediatric Urology, University Medical Center Mainz \\ (Johannes Gutenberg-University Mainz), 55131 Mainz, Germany \\ 6 German Center for Cardiovascular Research (DZHK), Partner Site Rhine Main, 55131 Mainz, Germany \\ * Correspondence: Karsten.Keller@unimedizin-mainz.de
}

check for updates

Citation: Keller, K.; Wöllner, J.; Schmitt, V.H.; Ostad, M.A.; Sagoschen, I.; Münzel, T.;

Espinola-Klein, C.; Hobohm, L. Risk Factors for Pulmonary Embolism in Patients with Paralysis and Deep Venous Thrombosis. J. Clin. Med. 2021, 10, 5412. https://doi.org/ $10.3390 /$ jcm10225412

Academic Editor: Sacha Zeerleder

Received: 5 October 2021

Accepted: 15 November 2021

Published: 19 November 2021

Publisher's Note: MDPI stays neutral with regard to jurisdictional claims in published maps and institutional affiliations.

Copyright: (c) 2021 by the authors. Licensee MDPI, Basel, Switzerland. This article is an open access article distributed under the terms and conditions of the Creative Commons Attribution (CC BY) license (https:/ / creativecommons.org/licenses/by/ $4.0 /)$.

\begin{abstract}
Background. Venous thromboembolism is a frequent complication and an important cause of death in patients with paralysis. We aimed to investigate predictors of pulmonary embolism (PE) and the impact of PE on the survival of patients with paralysis in comparison to those with deep venous thrombosis or thrombophlebitis (DVT). Methods: Patients were selected by screening the German nationwide inpatient sample (2005-2017) for paralysis, and were stratified for venous thromboembolism (VTE) and the VTE-sub-entity PE (ICD-code I26). Impact of PE on mortality and predictors for PE were analyzed. Results: Overall, 7,873,769 hospitalizations of patients with paralysis were recorded in Germany 2005-2017, of whom 1.6\% had VTE and 7.0\% died. While annual hospitalizations increased (2005: 520,357 to 2017: 663,998) ( $\beta 12,421$ (95\% CI 10,807 to 14,034), $p<0.001)$, in-hospital mortality decreased from $7.5 \%$ to $6.7 \%(\beta-0.08 \%(95 \% \mathrm{CI}-0.10 \%$ to $-0.06 \%)$, $p<0.001)$. When focusing on 82,558 patients with paralysis hospitalized due to VTE $(51.8 \%$ females; $58.3 \%$ aged $\geq 70$ years) in 2005-2017, in-hospital mortality was significantly higher in patients with paralysis and PE than in those with DVT only $(23.8 \%$ vs. $6.3 \%, p<0.001)$. Cancer (OR $2.18(95 \%$ CI 2.09-2.27), $p<0.001$ ), heart failure (OR 1.83 (95\% CI 1.76-1.91), $p<0.001$ ), COPD (OR 1.63 (95\% CI $1.53-1.72), p<0.001$ ) and obesity (OR 1.42 (95\% CI 1.35-1.50), $p<0.001$ ) were associated with PE. PE (OR 4.28 (95\% CI 4.07-4.50), $p<0.001$ ) was a strong predictor of in-hospital mortality. Conclusions: In Germany, annual hospitalizations of patients with paralysis increased in 2005-2017, in whom VTE and especially PE substantially affected in-hospital mortality. Cancer, heart failure, COPD, obesity and acute paraplegia were risk factors of PE.
\end{abstract}

Keywords: paralysis; stroke; mortality; spinal cord injury; pulmonary embolism

\section{Introduction}

Paralysis is categorized as a moderate to strong risk factor for the development of venous thromboembolism (VTE) [1-9].It has been estimated that the incidence of deep venous thrombosis in paralysis and stroke patients exceeds even the incidence of deep venous thrombosis in patients with general surgeries, and might be similar to the high rates occurring after hip and knee joint arthroplasty $[1,3,5-7,10,11]$. 
Without VTE prophylaxis, several studies revealed that up to $75 \%$ of the stroke patients with hemiplegia or paralysis develop deep venous thrombosis. $[1,5,6]$ Overall, two-thirds of these deep venous thrombosis events are asymptomatic and occur below the knee level [1]. Importantly, the risk for deep venous thrombosis correlated with the degree of paralysis, with predilection for the paralyzed leg [1,6].

In about $65 \%$ of all cases, pulmonary embolism (PE) is the consequence of deep venous thrombosis, rather than a separate clinical disease entity [12-14]. For PE without underlying deep venous thrombosis (isolated PE), data from different studies suggest an important role of co-morbidities, including cancer [15], atrial fibrillation [16,17], myocardial infarction [16] and heart failure $[16,17]$ in the pathogenesis of central thrombus formation. Although major trauma, surgery, immobilization, cancer and thrombophilia are well-recognized traditional risk factors for VTE $[9,18]$, several chronic inflammatory diseases, such as inflammatory bowel disease, psoriasis, rheumatoid arthritis, systemic lupus erythematosus, Sjogren syndrome, celiac disease, systemic sclerosis and dermatomyositis, as well as polymyositis, were associated with an increased risk of VTE [18-27].

Since the incidence of VTE, comprising deep venous thrombosis and PE, is high in patients with paralysis [3,5] and the VTE sub-entity PE, especially, is accompanied by a high risk of an adverse in-hospital course $[4,9,11,20]$ it is of outstanding interest to identify factors that result in or are associated with the development of the life-threatening complication of an acute PE.

\section{Methods and Patients}

For our present study, we analyzed the nationwide inpatient sample of Germany with diagnosis-related group (DRG) statistics. As implemented in the year 2004, patients' diagnoses must be coded in Germany according to the ICD-10-GM (International Classification of Diseases, 10th Revision with German Modification) and patients' diagnostical as well as surgical and interventional approaches must be coded according to OPS codes (Operationen- und Prozedurenschlüssel), and the hospitals send these codes to the Institute for the Hospital Remuneration System to obtain their remuneration. In parallel, the Federal Statistical Office of Germany (Statistisches Bundesamt) collects and assesses these data for the German nationwide inpatient sample, which was used for the present analysis. [7,28,29].

The Research Data Center (RDC) of the Federal Statistical Office and the Statistical Offices of the federal states in Wiesbaden (Germany) ran the study analyses on our behalf. For the trend analyses of hospitalizations of patients with paralysis (with and without VTE) 2005-2017, we requested from the RDC an analysis of total annual numbers of hospitalizations of patients with paralysis (ICD codes G80-G83), with and without VTE (ICD codes I26, I80-I82), and among these, deceased patients. Out of these data, rate of VTE and in-hospital mortality of hospitalized patients with paralysis were calculated (Statistisches Bundesamt, DEStatis, source: DRG-Statistik, Sonderauswertung des Statistischen Bundesamtes) [30].

For the second part of the statistical analysis, and therefore our further analysis in detail, we focused on an excerpt of these patients with paralysis in Germany and selected all hospitalized patients with paralysis (ICD codes G80-G83) in co-prevalence with VTE (main diagnosis of deep vein thrombosis or thrombophlebitis (DVT, ICD-codes I80, I81, I82) and/or main or secondary diagnosis of PE (ICD-code I26)) in Germany between 2005 and 2017; these patients were stratified for presence of PE (ICD-code I26) (source: RDC of the Federal Statistical Office and the Statistical Offices of the federal states, DRG Statistics 2005-2017, own calculations) (Figure 1). The main diagnosis of a patient was defined as the diagnosis mainly responsible for hospitalization documented by the discharging physician [31]. All analyzed VTE risk factors, comorbidities and adverse in-hospital events were defined and coded according to the ICD-10-GM. 


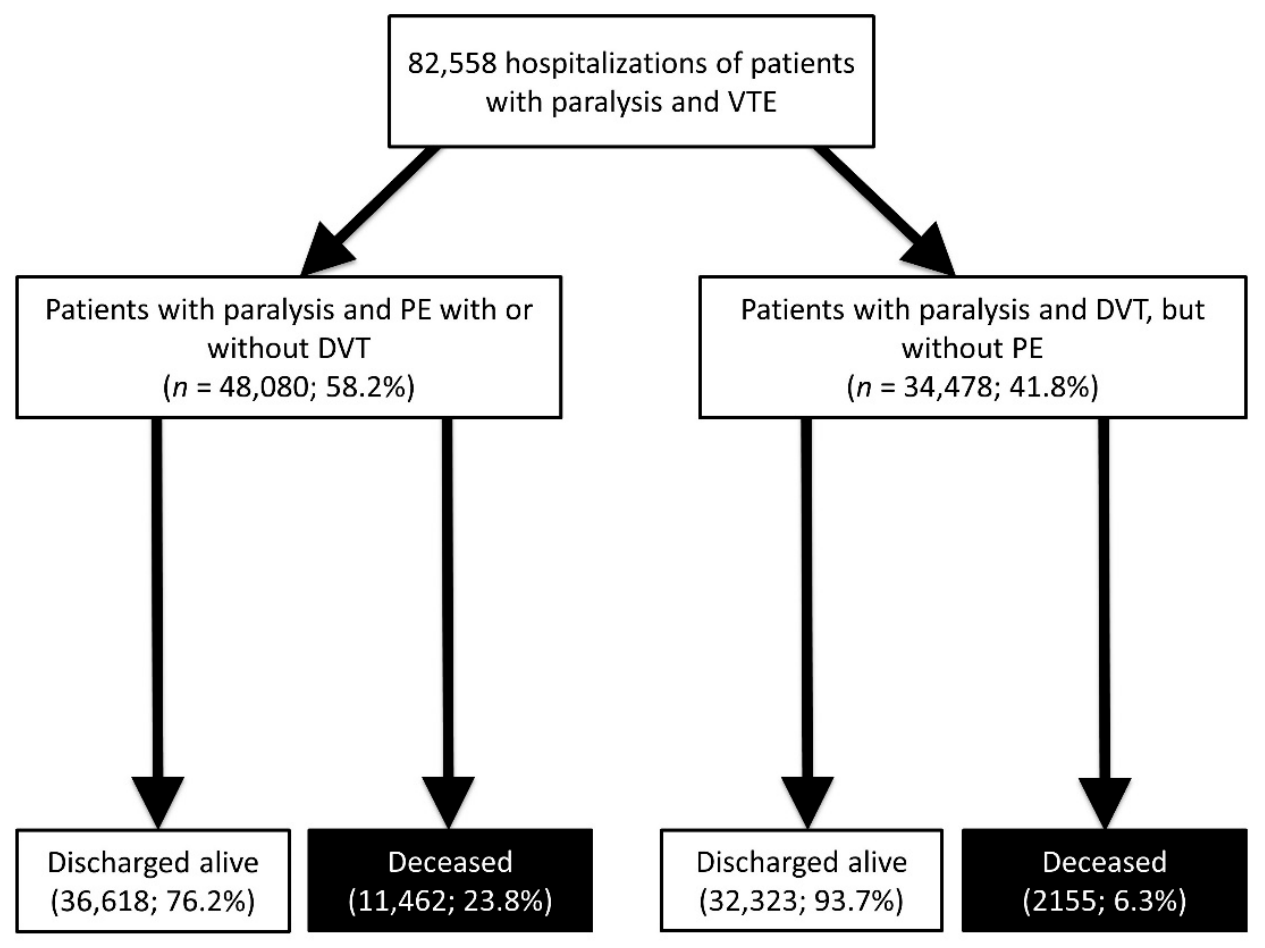

Figure 1. Flow diagram.

\subsection{Study Endpoints}

The primary study endpoints comprised (I) the presence of PE and (II) death from all causes during in-hospital stay (in-hospital death).

\subsection{Ethical Aspects and Study Oversight}

Since direct access by the investigators to the data of individual patients was not given in this present study, and only the access to summarized results provided by the RDC was the basis of these study results, approval by an ethics committee as well as an informed consent were not required, in accordance with German law [32].

\section{Statistics}

Descriptive statistics for the relevant comparisons of (i) patients with paralysis in co-prevalence with isolated DVT (without PE) and patients with paralysis in co-prevalence with PE (with or without DVT) and (ii) patients with paralysis in co-prevalence with VTE stratified for decease during hospitalization (in-hospital death) were shown as median and interquartile range (IQR), or presented as absolute numbers and corresponding percentages. In case of continuous variables, these variables/parameters were compared with the help of the Wilcoxon-Whitney U test, and categorical variables were tested with Fisher's exact or chi [2] test, as appropriate [32,33].

Logistic regression models were calculated to investigate (i) associations between patients' characteristics and adverse events, and presence of PE in patients with paralysis in co-prevalence with any VTE, in order to identify parameters that are accompanied by an association to PE in patients with paralysis and VTE, and (ii) associations between patients' characteristics and adverse events, as well as presence of PE and in-hospital death in patients with paralysis in co-prevalence with VTE. These results of the logistic regressions were presented as Odds Ratios (OR) and 95\% confidence intervals (CI). To ensure that the results of the logistic regression analyses were not substantially influenced by biasing factors, and therefore guarantee a broad independence from different cofactors, we adjusted the multivariate logistic regression models with the following parameters: age, gender, cancer, coronary artery disease, heart failure, chronic obstructive pulmonary 
disease (COPD), arterial hypertension, acute and chronic kidney disease, diabetes mellitus, atrial fibrillation/flutter, smoking and hyperlipidaemia.

We worked and operated with the software SPSS ${ }^{\circledR}$ (IBM Corp. Released 2011. IBM SPSS Statistics for Windows, Version 20.0. IBM Corp: Armonk, NY, USA) for the computerized analysis. Only $p$ values of $<0.05$ (two-sided) were considered to be statistically significant.

\section{Results}

\subsection{Time-Trends Regarding Hospitalizations of Patients with Paralysis with and without VTE}

Overall, 7,873,769 hospitalizations of patients with paralysis were recorded in the German nationwide inpatient sample between 2005 and 2017. In total, 550,058 (7.0\%) patients died during the observational period. While the annual hospitalizations increased from 520,357 in the year 2005 to 663,998 in the year 2017 ( $\beta$ 12,421.12 per year [95\% CI $10,807.73$ to $14,034.51], p<0.001)$, the in-hospital mortality decreased from $7.5 \%$ in 2005 to $6.7 \%$ in 2017 ( $\beta-0.08 \%$ per year (95\% CI $-0.10 \%$ to $-0.06 \%), p<0.001$ ) (Figure $2 \mathrm{~A}$ ). In total, 127,766 hospitalizations of patients with paralysis were counted with VTE (1.6\% of all hospitalizations of all patients with paralysis). In parallel with the annual number of hospitalizations of patients with paralysis, the hospitalizations of patients with paralysis and VTE increased from 8360 in 2005 to 11,890 in 2017 ( $\beta 287.87$ per year (95\% CI 242.39 to 333.35), $p<0.001$ ). In contrast, the proportion of patients with paralysis and VTE was widely constant with a small uptrend (1.6\% in 2005 and $1.8 \%$ in 2017$)(\beta 0.014 \%(95 \%$ CI $0.004 \%$ to $0.023 \%$ ), $p=0.008$ ) (Figure $2 \mathrm{~B}$ ). The in-hospital death rate was $13.5 \%$ in patients with paralysis and VTE, and considerably higher than in patients with paralysis only (in-hospital death rate: $7.0 \%$ ). Encouragingly, the in-hospital death rate of patients with paralysis and VTE decreased from $14.8 \%$ in the year 2005 to $12.9 \%$ in 2017 ( $\beta-0.15 \%$ (95\% CI $-0.19 \%$ to $-0.11 \%), p<0.001$ ) (Figure $2 \mathrm{~B}$ ).
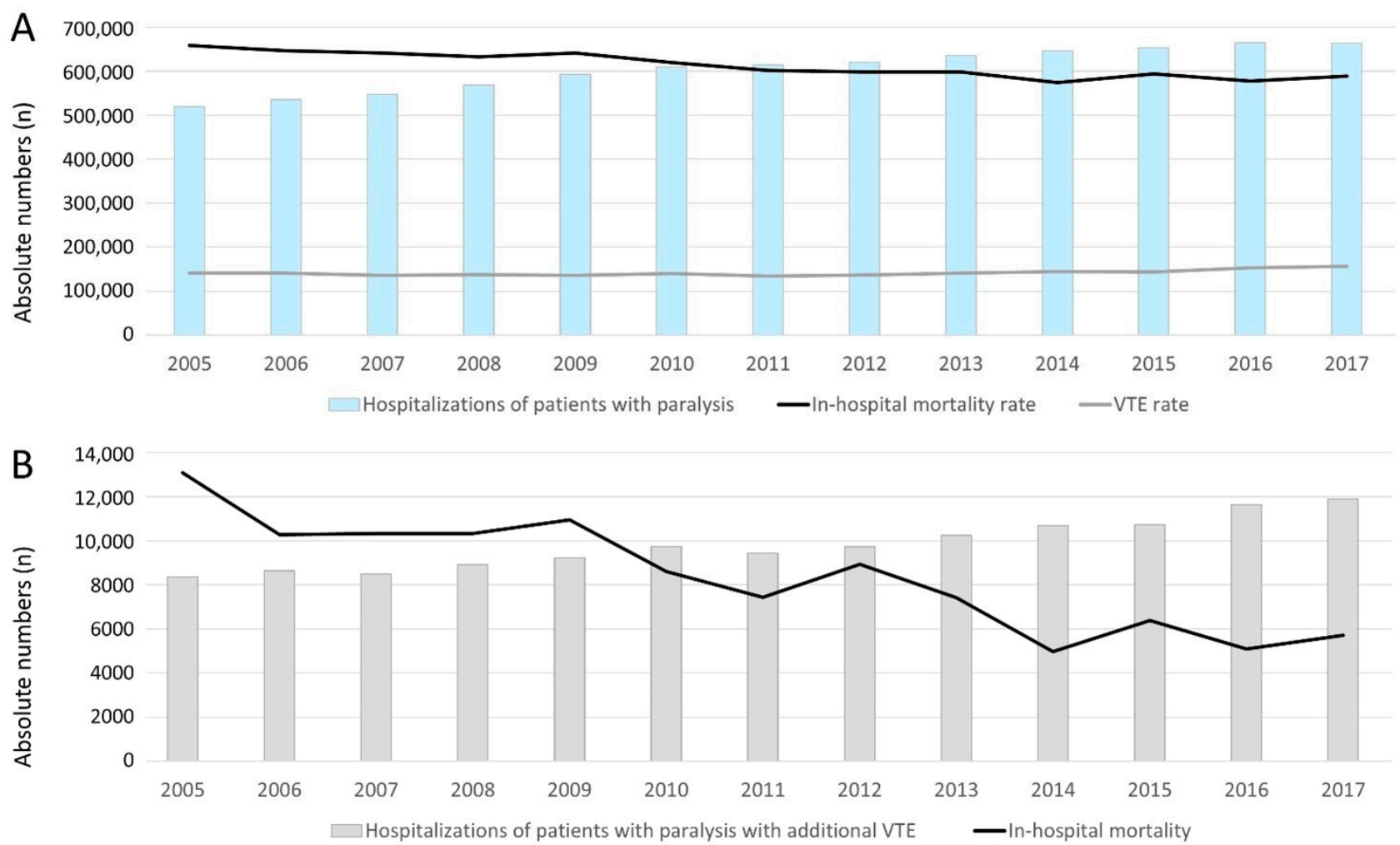

Figure 2. Temporal trends regarding hospitalizations of patients with paralysis in 2015-2017 in Germany (A): All hospitalizations of patients with paralysis (blue bars), VTE rate (grey line) and in-hospital death rate (black line). (B): Annual numbers of patients with paralysis and VTE (grey bars) and their in-hospital mortality rate (black line). 
4.2. Comparison of Patients with Paralysis in Co-Prevalence with Isolated DVT (without PE) and Patients with Paralysis in Co-Prevalence with PE (with or without DVT)

The German nationwide inpatient sample comprised 82,558 patients with paralysis hospitalized due to DVT or with PE (51.8\% females; 58.3\% aged $\geq 70$ years) between 2005 and 2017. Among these patients with paralysis, 48,080 (58.2\%) were diagnosed with PE and 34,478 patients $(41.8 \%)$ with isolated DVT (Figure 1). In total, 13,617 (16.5\%) of the patients with paralysis and VTE died during hospitalization (Figure 1).

Patients with paralysis and PE were slightly younger than those with paralysis and isolated DVT. The classical VTE risk factors, cancer and surgery, were substantially more common in patients with PE (Table 1). In addition, comorbidities, such as heart failure and COPD, were more prevalent in patients with paralysis and PE in comparison to those with paralysis and isolated DVT. In contrast, peripheral artery disease, arterial hypertension and hyperlipidaemia were more frequent in patients with paralysis and DVT only, and the groups did not differ regarding inflammatory bowel disease.

Table 1. Patients' characteristics, medical history, presentation, treatments and adverse in-hospital events of 82,558 patients with paralysis (ICD codes G80-G83) in co-prevalence with isolated DVT (without PE) (ICD-codes I26, I80, I81, I82) in comparison to patients with paralysis and PE (with or without DVT) (ICD-code I26).

\begin{tabular}{|c|c|c|c|}
\hline Parameters & $\begin{array}{l}\text { Patients with Paralysis and } \\
\text { PE with or without DVT } \\
(n=48,080 ; 58.2 \%)\end{array}$ & $\begin{array}{l}\text { Patients with Paralysis and } \\
\text { Isolated DVT (without PE) } \\
\quad(n=34,478 ; 41.8 \%)\end{array}$ & $p$-Value \\
\hline Median age (years) & $73.00(62.00-80.00)$ & $74.00(63.00-81.00)$ & $<0.001$ \\
\hline Age $\geq 70$ years & $27,337(56.9 \%)$ & $20,781(60.3 \%)$ & $<0.001$ \\
\hline Gender (females) & $24,520(51.0 \%)$ & $18,244(52.9 \%)$ & $<0.001$ \\
\hline In-hospital stay (days) & $15(8-28)$ & $12(7-22)$ & $<0.001$ \\
\hline Obesity & $4586(9.5 \%)$ & $2465(7.1 \%)$ & $<0.001$ \\
\hline \multicolumn{4}{|l|}{ Acute paraplegia and infantile cerebral palsy } \\
\hline Acute complete or incomplete paraplegia & $1337(2.8 \%)$ & $88(0.3 \%)$ & $<0.001$ \\
\hline Acute complete paraplegia & $354(0.7 \%)$ & $29(0.1 \%)$ & $<0.001$ \\
\hline Acute incomplete paraplegia & $1002(2.1 \%)$ & $60(0.2 \%)$ & $<0.001$ \\
\hline Infantile cerebral palsy & $346(0.7 \%)$ & $312(0.9 \%)$ & 0.003 \\
\hline \multicolumn{4}{|l|}{ VTE risk factors } \\
\hline Surgery during in-hospital stay & $30,237(62.9 \%)$ & $18,188(52.8 \%)$ & $<0.001$ \\
\hline Cancer & $9589(19.9 \%)$ & $3509(10.2 \%)$ & $<0.001$ \\
\hline Inflammatory bowel disease & $273(0.6 \%)$ & $189(0.5 \%)$ & 0.710 \\
\hline Thrombophilia & $411(0.8 \%)$ & $271(0.8 \%)$ & 0.282 \\
\hline \multicolumn{4}{|l|}{ Comorbidities } \\
\hline Heart failure & $11,512(23.9 \%)$ & $5162(14.9 \%)$ & $<0.001$ \\
\hline Coronary artery disease & $7479(15.6 \%)$ & $5198(15.1 \%)$ & 0.060 \\
\hline Cardiomyopathy & $711(1.5 \%)$ & $385(1.1 \%)$ & $<0.001$ \\
\hline Peripheral artery disease & $1994(4.1 \%)$ & $1814(5.3 \%)$ & $<0.001$ \\
\hline Chronic obstructive pulmonary disease & $4334(9.0 \%)$ & $1923(5.6 \%)$ & $<0.001$ \\
\hline Arterial hypertension & $24,310(50.6 \%)$ & $19,732(57.2 \%)$ & $<0.001$ \\
\hline Hyperlipidaemia & $7731(16.1 \%)$ & $8174(23.7 \%)$ & $<0.001$ \\
\hline Acute and chronic kidney disease & $11,091(23.1 \%)$ & $6459(18.7 \%)$ & $<0.001$ \\
\hline Diabetes mellitus & $12,468(25.9 \%)$ & $9141(26.5 \%)$ & 0.061 \\
\hline Deep venous thrombosis or thrombophlebitis & $14,224(29.6 \%)$ & $34,478(100.0 \%)$ & $<0.001$ \\
\hline Atrial fibrillation/flutter & $11,775(24.5 \%)$ & $8568(24.9 \%)$ & 0.236 \\
\hline \multicolumn{4}{|l|}{ Risk stratification markers } \\
\hline Right ventricular dysfunction & $14,702(30.6 \%)$ & $0(0.0 \%)$ & $<0.001$ \\
\hline Syncope & $876(1.8 \%)$ & $255(0.7 \%)$ & $<0.001$ \\
\hline
\end{tabular}


Table 1. Cont.

\begin{tabular}{|c|c|c|c|}
\hline Parameters & $\begin{array}{l}\text { Patients with Paralysis and } \\
\text { PE with or without DVT } \\
(n=48,080 ; 58.2 \%)\end{array}$ & $\begin{array}{l}\text { Patients with Paralysis and } \\
\text { Isolated DVT (without PE) } \\
\quad(n=34,478 ; 41.8 \%)\end{array}$ & $p$-Value \\
\hline \multicolumn{4}{|l|}{ Adverse events during the in-hospital stay } \\
\hline All cause in-hospital death & $11,462(23.8 \%)$ & $2155(6.3 \%)$ & $<0.001$ \\
\hline Stroke & $16,456(34.2 \%)$ & $20,617(59.8 \%)$ & $<0.001$ \\
\hline Myocardial injury & $1742(3.6 \%)$ & $768(2.2 \%)$ & $<0.001$ \\
\hline Shock & $2611(5.4 \%)$ & $520(1.5 \%)$ & $<0.001$ \\
\hline Cardio-pulmonary resuscitation & $4087(8.5 \%)$ & $329(1.0 \%)$ & $<0.001$ \\
\hline Intracerebral bleeding & $3442(7.1 \%)$ & $1094(3.2 \%)$ & $<0.001$ \\
\hline Subarachnoid hemorrhage & $592(1.2 \%)$ & $462(1.3 \%)$ & 0.169 \\
\hline Gastro-intestinal bleeding & $1026(2.1 \%)$ & $469(1.4 \%)$ & $<0.001$ \\
\hline \multicolumn{4}{|l|}{$\begin{array}{l}\text { Reperfusion treatments and transfusion of } \\
\text { blood constituents }\end{array}$} \\
\hline Systemic thrombolysis & $3086(6.4 \%)$ & $2174(6.3 \%)$ & 0.512 \\
\hline Transfusion of blood constituents & $8679(18.1 \%)$ & $3202(9.3 \%)$ & $<0.001$ \\
\hline
\end{tabular}

Abbreviations: VTE indicates venous thromboembolism, PE = pulmonary embolism, DVT = deep venous thrombosis or thrombophlebitis.

While the paralysis was related to an acute stroke event in $59.8 \%(20,617)$ of the patients with co-prevalence of paralysis with isolated DVT, stroke was substantially less prevalent in patients with paralysis and PE, at $34.2 \%(16,456)(p<0.001)$. While the acute paraplegia sub-entities were more prevalent in patients with paralysis and PE than in those with DVT only, infantile cerebral palsy was more frequent in patients with paralysis and DVT only, compared to those with PE (Table 1).

As expected, the risk stratification markers of right ventricular dysfunction (RVD), myocardial injury, syncope and shock occurred more often in patients with PE compared to those with DVT but without PE (Table 1).

All-cause in-hospital mortality was significantly higher in patients with paralysis and PE than in those with DVT only $(23.8 \%$ vs. $6.3 \%, p<0.001)$. In line, bleeding events, such as intracerebral bleeding, gastro-intestinal bleeding and the necessity for transfusion of blood constituents were higher in patients with paralysis and PE (Table 1).

\subsection{Risk Factors for Occurrence of PE in Patients with Paralysis and VTE}

All investigated acute forms of paraplegia, such as acute complete or incomplete paraplegia (OR 9.80 (95\% CI 7.89-12.18), $p<0.001$ ), acute complete paraplegia (OR 7.55 (95\% CI 5.15-11.06), $p<0.001$ ) and acute incomplete paraplegia were strongly associated with PE (OR 10.77 (95\% CI 8.29-14.01), $p<0.001$ ) (Table 2). Cancer (OR 2.18 (95\% CI 2.092.27), $p<0.001$ ), heart failure (OR 1.83 (95\% CI 1.76-1.91), $p<0.001$ ), COPD (OR $1.63(95 \%$ CI 1.53-1.72), $p<0.001$ ) and obesity (OR 1.42 (95\% CI 1.35-1.50), $p<0.001$ ) were important and independent risk factors for a PE in patients with paralysis (and VTE) (Table 2). In contrast, peripheral artery disease, arterial hypertension and hyperlipidaemia, as well as stroke, were independently associated with paralysis in co-prevalence with DVT but without PE (Table 2).

Table 2. Factors associated with pulmonary embolism in patients with paralysis in coprevalence with venous thromboembolism.

\begin{tabular}{ccccc}
\hline & \multicolumn{2}{c}{ Uni-Variate Regression Model } & \multicolumn{2}{c}{ Multi-Variate Regression Model } \\
\hline Parameters & OR (95\% CI) & $p$-Value & OR (95\% CI) & $p$-Value \\
\hline Age & $0.995(0.994-0.996)$ & $<0.001$ & $0.994(0.993-0.996)$ & $<0.001$ \\
Age $\geq 70$ years & $0.87(0.85-0.89)$ & $<0.001$ & $0.87(0.84-0.90)$ & $<0.001$ \\
Gender (females) & $0.93(0.90-0.95)$ & $<0.001$ & $0.96(0.94-0.99)$ & 0.011 \\
Obesity & $1.37(1.30-1.44)$ & $<0.001$ & $1.42(1.35-1.50)$ & $<0.001$ \\
\hline
\end{tabular}


Table 2. Cont.

\begin{tabular}{|c|c|c|c|c|}
\hline \multirow[b]{2}{*}{ Parameters } & \multicolumn{2}{|c|}{ Uni-Variate Regression Model } & \multicolumn{2}{|c|}{ Multi-Variate Regression Model } \\
\hline & OR $(95 \%$ CI) & $p$-Value & OR $(95 \% \mathrm{CI})$ & $p$-Value \\
\hline \multicolumn{5}{|l|}{$\begin{array}{l}\text { Acute paraplegia and infantile } \\
\text { cerebral Palsy }\end{array}$} \\
\hline Acute complete or incomplete paraplegia & $11.18(9.00-13.87)$ & $<0.001$ & $9.80(7.89-12.18)$ & $<0.001$ \\
\hline Acute complete paraplegia & $8.81(6.03-12.87)$ & $<0.001$ & $7.55(5.15-11.06)$ & $<0.001$ \\
\hline Acute incomplete paraplegia & $12.21(9.40-15.84)$ & $<0.001$ & $10.77(8.29-14.01)$ & $<0.001$ \\
\hline Infantile cerebral palsy & $0.79(0.68-0.93)$ & 0.003 & $0.73(0.62-0.85)$ & $<0.001$ \\
\hline \multicolumn{5}{|l|}{ VTE risk factors } \\
\hline Surgery during in-hospital stay & $1.52(1.48-1.56)$ & $<0.001$ & $1.44(1.40-1.48)$ & $<0.001$ \\
\hline Cancer & $2.20(2.11-2.29)$ & $<0.001$ & $2.18(2.09-2.27)$ & $<0.001$ \\
\hline Inflammatory bowel disease & $1.04(0.86-1.25)$ & 0.710 & $0.99(0.82-1.20)$ & 0.918 \\
\hline Thrombophilia & $1.09(0.93-1.27)$ & 0.282 & $1.04(0.89-1.22)$ & 0.642 \\
\hline \multicolumn{5}{|l|}{ Comorbidities } \\
\hline Heart failure & $1.79(1.72-1.85)$ & $<0.001$ & $1.83(1.76-1.91)$ & $<0.001$ \\
\hline Coronary artery disease & $1.04(1.00-1.08)$ & 0.060 & $1.01(0.97-1.05)$ & 0.617 \\
\hline Cardiomyopathy & $1.33(1.17-1.51)$ & $<0.001$ & $1.06(0.93-1.20)$ & 0.414 \\
\hline Chronic obstructive pulmonary disease & $1.68(1.59-1.77)$ & $<0.001$ & $1.63(1.53-1.72)$ & $<0.001$ \\
\hline Hyperlipidaemia & $0.62(0.60-0.64)$ & $<0.001$ & $0.65(0.63-0.68)$ & $<0.001$ \\
\hline Arterial hypertension & $0.76(0.74-0.79)$ & $<0.001$ & $0.88(0.86-0.91)$ & $<0.001$ \\
\hline Acute and chronic kidney disease & $1.30(1.26-1.35)$ & $<0.001$ & $1.24(1.20-1.29)$ & $<0.001$ \\
\hline Diabetes mellitus & $0.97(0.94-1.00)$ & 0.061 & $0.97(0.94-1.01)$ & 0.123 \\
\hline Peripheral artery disease & $0.78(0.73-0.83)$ & $<0.001$ & $0.75(0.70-0.80)$ & $<0.001$ \\
\hline Atrial fibrillation/flutter & $0.98(0.95-1.01)$ & 0.236 & $0.96(0.93-1.00)$ & 0.034 \\
\hline Stroke (ischemic and hemorrhagic) & $0.35(0.34-0.36)$ & $<0.001$ & $0.36(0.35-0.37)$ & $<0.001$ \\
\hline
\end{tabular}

4.4. Differences between Patients with Paralysis and VTE Who Died during Hospitalization and Those Who Were Discharged Alive

Overall, 13,617 (16.5\%) patients with paralysis and VTE died during their in-hospital stay. The deceased patients with paralysis and VTE were in median 2 years older and more often had cancer, heart failure, coronary artery disease, COPD, acute and chronic kidney disease and diabetes mellitus than those who were discharged alive (Table 3). PE was substantially more common in deceased patients $(84.2 \%$ vs. $53.1 \%, p<0.001)$. Bleeding events, such as intracerebral bleeding, gastro-intestinal bleeding and necessity of transfusion of blood constituents, as well as shock, were more frequent in patients who died during the in-hospital course (Table 3).

Table 3. Characteristics, medical history, presentation, treatments and adverse in-hospital events of 82,558 patients with paralysis (ICD codes G80-G83) in co-prevalence with venous thromboembolism (ICD-codes I26, I80, I81, I82) and stratified for in-hospital death.

\begin{tabular}{|c|c|c|c|}
\hline Parameters & $\begin{array}{l}\text { Patients with Paralysis and } \\
\text { VTE, Who Died IN-Hospital } \\
(n=13,617 ; 16.5 \%)\end{array}$ & $\begin{array}{l}\text { Patients with Paralysis and VTE, } \\
\text { Who Were Discharged Alive } \\
(n=68,941 ; 83.5 \%)\end{array}$ & $p$-Value \\
\hline Median age (years) & $75.0(66.0-82.0)$ & $73.0(62.0-80.0)$ & $<0.001$ \\
\hline Age $\geq 70$ years & $8801(64.6 \%)$ & $39,317(57.0 \%)$ & $<0.001$ \\
\hline Gender (females) & $6953(51.1 \%)$ & $35,811(51.9 \%)$ & 0.059 \\
\hline In-hospital stay (days) & $10(4-21)$ & $15(8-27)$ & $<0.001$ \\
\hline Obesity & $997(7.3 \%)$ & $6054(8.8 \%)$ & $<0.001$ \\
\hline \multicolumn{4}{|l|}{ Paraplegia and infantile cerebral palsy } \\
\hline Acute complete or incomplete paraplegia & $255(1.9 \%)$ & $1170(1.7 \%)$ & 0.141 \\
\hline Acute complete paraplegia & $85(0.6 \%)$ & $298(0.4 \%)$ & 0.002 \\
\hline Acute incomplete paraplegia & $173(1.3 \%)$ & $889(1.3 \%)$ & 0.880 \\
\hline Infantile cerebral palsy & $95(0.7 \%)$ & $563(0.8 \%)$ & 0.160 \\
\hline
\end{tabular}


Table 3. Cont.

\begin{tabular}{|c|c|c|c|}
\hline Parameters & $\begin{array}{l}\text { Patients with Paralysis and } \\
\text { VTE, Who Died IN-Hospital } \\
(n=13,617 ; 16.5 \%)\end{array}$ & $\begin{array}{l}\text { Patients with Paralysis and VTE, } \\
\text { Who Were Discharged Alive } \\
(n=68,941 ; 83.5 \%)\end{array}$ & $p$-Value \\
\hline \multicolumn{4}{|l|}{ VTE risk factors } \\
\hline Surgery during in-hospital stay & $7635(56.1 \%)$ & $40,790(59.2 \%)$ & $<0.001$ \\
\hline Cancer & $3340(24.5 \%)$ & $9758(14.2 \%)$ & $<0.001$ \\
\hline Inflammatory bowel disease & $79(0.6 \%)$ & $383(0.6 \%)$ & 0.710 \\
\hline Thrombophilia & $78(0.6 \%)$ & $604(0.9 \%)$ & $<0.001$ \\
\hline \multicolumn{4}{|l|}{ Comorbidities } \\
\hline Heart failure & $3926(28.7 \%)$ & $12,748(18.4 \%)$ & $<0.001$ \\
\hline Coronary artery disease & $2358(17.3 \%)$ & $10,319(15.0 \%)$ & $<0.001$ \\
\hline Cardiomyopathy & $230(1.7 \%)$ & $866(1.2 \%)$ & $<0.001$ \\
\hline Chronic obstructive pulmonary disease & $1298(9.5 \%)$ & $4959(7.2 \%)$ & $<0.001$ \\
\hline Arterial hypertension & $6420(47.1 \%)$ & $37,622(54.6 \%)$ & $<0.001$ \\
\hline Hyperlipidaemia & $1732(12.7 \%)$ & $14,173(20.6 \%)$ & $<0.001$ \\
\hline Acute and chronic kidney disease & $4205(30.9 \%)$ & $13,345(19.4 \%)$ & $<0.001$ \\
\hline Diabetes mellitus & $3995(29.3 \%)$ & $17,614(25.5 \%)$ & $<0.001$ \\
\hline Pulmonary embolism & $11,462(84.2 \%)$ & $36,618(53.1 \%)$ & $<0.001$ \\
\hline Deep venous thrombosis or thrombophlebitis & $4029(29.6 \%)$ & $44,673(64.8 \%)$ & $<0.001$ \\
\hline Stroke & $6560(48.2 \%)$ & $30,513(44.3 \%)$ & $<0.001$ \\
\hline Myocardial injury & $727(5.3 \%)$ & $1783(2.6 \%)$ & $<0.001$ \\
\hline Atrial fibrillation/flutter & $4026(29.6 \%)$ & $16,317(23.7 \%)$ & $<0.001$ \\
\hline Intracerebral bleeding & $1034(7.6 \%)$ & $3502(5.0 \%)$ & $<0.001$ \\
\hline Subarachnoid hemorrhage & $170(1.2 \%)$ & $884(1.3 \%)$ & 0.770 \\
\hline Gastro-intestinal bleeding & $411(3.0 \%)$ & $1084(1.6 \%)$ & $<0.001$ \\
\hline Transfusion of blood constituents & $3233(23.7 \%)$ & $8648(12.5 \%)$ & $<0.001$ \\
\hline Shock & $1557(11.4 \%)$ & $1574(2.3 \%)$ & $<0.001$ \\
\hline
\end{tabular}

Abbreviations: VTE indicates venous thromboembolism, PE = pulmonary embolism, DVT = deep venous thrombosis or thrombophlebitis.

\subsection{Predictors of In-Hospital Death in Patients with Paralysis and VTE}

PE (OR 4.28 (95\% CI 4.07-4.50), $p<0.001)$ and RVD (OR 4.84 (95\% CI 4.64-5.05), $p<0.001)$ as well as shock (OR 5.06 (95\% CI 4.69-5.46), $p<0.001$ were the strongest independent PE-specific predictors of in-hospital mortality in patients with paralysis and VTE (Table 4). Age $\geq 70$ years (OR 1.35 (95\% CI 1.29-1.41), $p<0.001)$, cancer (OR 2.18 (95\% CI 2.08-2.28), $p<0.001$ ), heart failure (OR 1.50 (95\% CI 1.43-1.57), $p<0.001$ ), acute and chronic kidney disease (OR 1.62 (95\% CI 1.55-1.69), $p<0.001)$, stroke (OR 1.32 (95\% CI 1.27-1.37), $p<0.001)$ and all bleeding events were independently associated with increased mortality (Table 4).

Table 4. Risk factors for in-hospital death in patients with paralysis in co-prevalence with venous thromboembolism.

\begin{tabular}{|c|c|c|c|c|}
\hline \multirow[b]{2}{*}{ Parameters } & \multicolumn{2}{|c|}{ Uni-Variate Regression Model } & \multicolumn{2}{|c|}{ Multi-Variate Regression Model } \\
\hline & OR $(95 \% \mathrm{CI})$ & $p$-Value & OR $(95 \% \mathrm{CI})$ & $p$-Value \\
\hline Age & $1.016(1.015-1.017)$ & $<0.001$ & $1.016(1.014-1.017)$ & $<0.001$ \\
\hline Age $\geq 70$ years & $1.38(1.33-1.43)$ & $<0.001$ & $1.35(1.29-1.41)$ & $<0.001$ \\
\hline Gender (females) & $0.97(0.93-1.00)$ & 0.059 & $0.90(0.87-0.94)$ & $<0.001$ \\
\hline Obesity & $0.82(0.77-0.88)$ & $<0.001$ & $0.89(0.82-0.95)$ & 0.001 \\
\hline \multicolumn{5}{|l|}{ Paraplegia and infantile cerebral palsy } \\
\hline Acute complete or incomplete paraplegia & $1.11(0.97-1.27)$ & 0.141 & $0.99(0.86-1.14)$ & 0.987 \\
\hline Acute complete paraplegia & $1.45(1.14-1.85)$ & 0.003 & $1.31(1.02-1.68)$ & 0.037 \\
\hline Acute incomplete paraplegia & $0.99(0.84-1.16)$ & 0.880 & $0.88(0.74-1.04)$ & 0.129 \\
\hline Infantile cerebral palsy & $0.86(0.69-1.06)$ & 0.160 & $1.25(1.00-1.57)$ & 0.050 \\
\hline
\end{tabular}


Table 4. Cont.

\begin{tabular}{|c|c|c|c|c|}
\hline \multirow[b]{2}{*}{ Parameters } & \multicolumn{2}{|c|}{ Uni-Variate Regression Model } & \multicolumn{2}{|c|}{ Multi-Variate Regression Model } \\
\hline & OR $(95 \% \mathrm{CI})$ & $p$-Value & OR $(95 \% \mathrm{CI})$ & $p$-Value \\
\hline \multicolumn{5}{|l|}{ VTE risk factors } \\
\hline Surgery during in-hospital stay & $0.88(0.85-0.91)$ & $<0.001$ & $0.83(0.80-0.86)$ & $<0.001$ \\
\hline Cancer & $1.97(1.89-2.06)$ & $<0.001$ & $2.18(2.08-2.28)$ & $<0.001$ \\
\hline Inflammatory bowel disease & $1.05(0.82-1.34)$ & 0.710 & $1.18(0.92-1.51)$ & 0.199 \\
\hline Thrombophilia & $0.65(0.52-0.83)$ & $<0.001$ & $0.78(0.61-0.99)$ & 0.043 \\
\hline \multicolumn{5}{|l|}{ Comorbidities } \\
\hline Heart failure & $1.79(1.72-1.87)$ & $<0.001$ & $1.50(1.43-1.57)$ & $<0.001$ \\
\hline Coronary artery disease & $1.19(1.13-1.25)$ & $<0.001$ & $1.04(0.98-1.09)$ & 0.185 \\
\hline Cardiomyopathy & 1.35 (1.17-1.57) & $<0.001$ & $1.17(1.00-1.36)$ & 0.048 \\
\hline Chronic obstructive pulmonary disease & $1.36(1.28-1.45)$ & $<0.001$ & $1.21(1.13-1.29)$ & $<0.001$ \\
\hline Hyperlipidaemia & $0.56(0.53-0.59)$ & $<0.001$ & $0.58(0.54-0.61)$ & $<0.001$ \\
\hline Arterial hypertension & $0.74(0.72-0.77)$ & $<0.001$ & $0.76(0.73-0.79)$ & $<0.001$ \\
\hline Acute and chronic kidney disease & $1.86(1.79-1.94)$ & $<0.001$ & $1.62(1.55-1.69)$ & $<0.001$ \\
\hline Diabetes mellitus & $1.21(1.16-1.26)$ & $<0.001$ & $1.13(1.09-1.18)$ & $<0.001$ \\
\hline Pulmonary embolism & $4.70(4.47-4.93)$ & $<0.001$ & $4.28(4.07-4.50)$ & $<0.001$ \\
\hline Peripheral artery disease & $1.46(1.35-1.58)$ & $<0.001$ & $1.31(1.21-1.43)$ & $<0.001$ \\
\hline Atrial fibrillation/flutter & $1.35(1.30-1.41)$ & $<0.001$ & $1.17(1.12-1.22)$ & $<0.001$ \\
\hline Stroke (ischemic and hemorrhagic) & $1.17(1.13-1.22)$ & $<0.001$ & $1.32(1.27-1.37)$ & $<0.001$ \\
\hline \multicolumn{5}{|l|}{ Risk stratification parameters } \\
\hline Syncope & $1.08(0.92-1.26)$ & 0.340 & $0.98(0.84-1.15)$ & 0.790 \\
\hline Right ventricular dysfunction in PE & $4.94(4.75-5.15)$ & $<0.001$ & $4.84(4.64-5.05)$ & $<0.001$ \\
\hline Shock & $5.46(5.08-5.87)$ & $<0.001$ & $5.06(4.69-5.46)$ & $<0.001$ \\
\hline \multicolumn{5}{|l|}{ Adverse in-hospital events } \\
\hline Intracerebral bleeding & $1.54(1.43-1.65)$ & $<0.001$ & $1.85(1.72-1.99)$ & $<0.001$ \\
\hline Subarachnoid bleeding & $0.98(0.83-1.15)$ & 0.770 & $1.39(1.17-1.64)$ & $<0.001$ \\
\hline Gastro-intestinal bleeding & $1.95(1.74-2.19)$ & $<0.001$ & $1.66(1.47-1.87)$ & $<0.001$ \\
\hline Transfusion of blood constituents & $2.16(2.07-2.26)$ & $<0.001$ & $1.90(1.81-1.99)$ & $<0.001$ \\
\hline
\end{tabular}

\section{Discussion}

The main results of our present study can be summarized as follows:

1. In total, $7,873,769$ hospitalizations of patients with paralysis were recorded in the German nationwide inpatient sample with an increase in the total annual number from 2005 to 2017.

2. Among these patients, $7.0 \%$ died during the observational period, and this rate decreased during the observational period.

3. Overall, $1.6 \%$ of all hospitalized patients with paralysis had or developed an event of VTE.

4. The in-hospital mortality rate was $13.5 \%$ in patients with paralysis and VTE.

5. Cancer, heart failure, COPD, and obesity were important and independent risk factors for PE in patients with paralysis and VTE, whereas peripheral artery disease, arterial hypertension and hyperlipidaemia as well as stroke were independently associated with paralysis in co-prevalence with DVT only.

6. Acute forms of paraplegia were strongly associated with PE in paralysis.

7. Independent predictors of in-hospital death in patients with paralysis and VTE were $P E$, shock, age $\geq 70$ years, cancer, heart failure, acute and chronic kidney disease, stroke as well as all bleeding events.

\subsection{Prevalence of Hospitalizations of Patients with Paralysis}

The prevalence of paralysis was estimated as $1.7 \%$ in the US population in the year 2013, which represents more than 5 million people in the US [34]. In accordance with those 
data, the German inpatient sample revealed a high number of hospitalizations of patients with paralysis $(7,873,769$ hospitalizations) in the observational period 2005-2017 as well. In Germany, the annual hospitalizations of patients with paralysis increased from 2005 to 2017, which is in accordance with an increasing number of disabling strokes based on the data of the Global Burden of Disease 2013 Study [35].

\subsection{Main Causes of Paralysis}

Stroke was the leading cause of paralysis in literature, followed by spinal cord injuries [34]. Remarkably, almost $72 \%$ of persons with paralysis were younger than 65 years [34]. Similarly, our study showed a high stroke rate (44.9\%) in patients with paralysis and VTE. Notably, stroke rate was lower in patients with paralysis and PE in comparison to those with paralysis and isolated DVT.

\subsection{Prevalence of VTE in Hospitalizations of Patients with Paralysis}

Paralysis is a moderate to strong risk factor for the development of VTE [8,9]. In patients with paralysis it is of particular interest that due to disabilities and immobilization, thromboembolic prophylaxis seems unfortunately to be less effective than in surgical patients [3].

Overall, $1.6 \%$ of all hospitalized patients with paralysis developed an event of VTE. As known from other studies, the prevalence of DVT (symptomatic and asymptomatic) differs in surgical and non-surgical medicine without VTE prophylaxis-between $10 \%$ and $20 \%$ in internal medicine diseases, distinctly higher in stroke (20-50\%) and polytrauma (40-80\%), and highest in patients with spinal cord injury $(60-80 \%)$ [36]. Other studies reported that without VTE prophylaxis, approximately $50-75 \%$ of stroke patients with hemiplegia or paralysis develop DVT $[1,5,6]$. Thus, VTE is a common complication in spinal cord injury and stroke with resulting paresis $[3,5,7-11,36]$. Among traumatic injuries, traumatic spine injuries of the spinal cord and/or the cauda equina are connected with the highest VTE risk [36]. Remarkably, the primary reasons for this high risk are related to the failure of the muscle pump driven by the paresis in combination with a presumed transient hypercoagulative phase, and, particularly, endothelial damage [36]. Moreover, decoupling from supraspinal control seems to be another important contributing prothrombotic factor [36].

Besides leg oedema caused by post-thrombotic syndrome and hemorrhage as a complication of the required anticoagulation, VTE in particular is related to poor outcome in patients with paralysis [36]. DVT is one of the major complications in patients with paralysis because of Virchow's triad, which comprises stasis, endothelial cell injury and hypercoagulability [37]. PE occurs in the large majority of cases when a thrombotic material from DVT breaks loose and travels through the bloodstream to the pulmonary artery bed [7,9,14], and is the most important and life-threatening manifestation of VTE [36]. Since DVT is the most common VTE entity in patients with paralysis, it is of outstanding interest that the majority of these DVT events is asymptomatic [1,12-14]. Thus, PE might be the first recognized manifestation of VTE, although the underlying previously developed DVT event might be undetected or overlooked, which can impede a life-saving early start to anticoagulant treatment $[1,12-14]$

\subsection{PE Is Related to a High Risk of In-Hospital Mortality in Patients with Paralysis}

Notably, in patients with stroke or paralysis, PE occurs in approximately one-fifth of these patients [5], and PE events are affected by aggravated outcomes with high morbidity and mortality $[5,7,9,32]$. Therefore, since the incidence of DVT is high in patients with paralysis [3,5] the risk for PE increases with DVT rate and PE events are accompanied by a high risk of death in the short-term $[4,9,11,20]$, it is of major interest to identify factors associated with development of the life-threatening complication of acute PE. 


\subsection{Predictors of PE in Patients with Paralysis}

Our study identified cancer, heart failure, COPD, obesity and acute and chronic kidney diseases as important and independent risk factors for the occurrence of PE in patients with paralysis and VTE. These findings are in broad agreement with previous study results reporting the important roles of cancer [15], atrial fibrillation $[16,17]$ myocardial infarction [16] and heart failure [16,17] in the pathogenesis of PE. In contrast, peripheral artery disease, arterial hypertension and hyperlipidaemia were independently associated with DVT without PE in patients with paralysis and VTE. In this context, a link between atherosclerosis and DVT was demonstrated in several studies [33,38-42]. Prandoni et al. reported a higher frequency of carotid plaques as an indicator of atherosclerosis in patients with previous idiopathic DVT in comparison to those without DVT [39]. Other studies confirmed the association between atherosclerosis and atherosclerotic risk factors, such as hyperlipidaemia and VTE [33,41-45]. Acute forms of paraplegia were strongly associated with PE in paralysis, which is in accordance with published literature [46].

\subsection{In-Hospital Mortality Rate of Patients with Paralysis}

The reported in-hospital mortality rate of patients hospitalized with paralysis was $7.0 \%$, and thus in line to other studies reporting in-hospital mortality rates due to acute stroke as between $5 \%$ and $14 \%$ [7,47-49], as well as death due to spinal cord injury ranging between $2 \%$ and $24 \%$ [50,51]. In this context, it is important to mention that the results of hospitalized patients with paralysis in our study do not focus only on these acute forms of paralysis, but also include patients with long-lasting non-acute forms of paralysis, which might explain the lower in-hospital mortality in our study.

\subsection{Impact of VTE on Survival of Patients with Paralysis}

As expected, VTE is a frequent and harmful complication in patients with paralysis [1-7], and the mortality rate in hospitalizations of patients with paralysis was substantially higher in presence of VTE. In addition, at $13.5 \%$, the in-hospital mortality rate in patients with paralysis and VTE was 1.9-fold higher than that of patients with paralysis (7.0\%) but without VTE.

\subsection{Independent Predictors of In-Hospital Death in Patients with Paralysis and VTE}

In accordance with the literature, we identified that higher age, cancer, heart failure, acute and chronic kidney disease, stroke, all bleeding events and PE are independent predictors of in-hospital death in patients with paralysis and VTE $[7,9,32,52-56]$. Notably, PE was associated with a 4.3-fold risk of death during hospitalization, which is in line with previous studies confirming the marked impact of PE and respiratory complications on in-hospital mortality [7,57-59].

In this context, it must also be considered that the number of PE-related deaths may even be underestimated in paralytic patients, since approximately half of the PE events in these patients occur as sudden death, and therefore might not be recognized as PE $[3,4,7]$.

\section{Limitations}

There are certain limitations of our present study which merit consideration: Firstly, as aforementioned, study results were based on ICD and OPS codes, which might be subject to underreporting or miscoding. Secondly, detailed baseline data, including patients' concomitant medications, cardiac troponin plasma concentrations, and echocardiographic parameters, and outcome data, such as major bleeding, were not available in the German nationwide inpatient sample. Third, exact timing of acute adverse events during hospitalizations could not be determined. Fourthly, information on the exact cause of death cannot be obtained from the German nationwide inpatient sample [32]. 


\section{Conclusions}

In Germany, the annual hospitalizations of patients with paralysis increased from 2005 to 2017. Among these inpatients, 7.0\% died and 1.6\% developed an event of VTE. VTE affected the in-hospital mortality rate of patients with paralysis substantially. Cancer, heart failure, COPD, obesity and acute paraplegia were important and independent risk factors for acute PE. PE was associated with 4.3-fold increase in-hospital mortality.

Author Contributions: Conceptualization, K.K. and L.H.; Data curation, K.K.; Formal analysis, K.K.; Investigation, K.K.; Methodology, K.K. and L.H.; Project administration, K.K.; Resources, K.K.; Supervision, K.K.; Validation, K.K.; Visualization, K.K.; Writing-original draft, K.K.; Writing-review \& editing, K.K., J.W., V.H.S., M.A.O., I.S., T.M., C.E.-K. and L.H. All authors have read and agreed to the published version of the manuscript.

Funding: This research received no external funding.

Institutional Review Board Statement: The study was conducted according to the guidelines of the Declaration of Helsinki. In accordance with German law, an approval by an ethical committee, institutional review board as well as in-formed consent of the patients were not required, since the present study did not involve a direct access by the study investigators on data of individual patients.

Informed Consent Statement: In accordance with German law, an approval by an ethical committee, institutional review board as well as in-formed consent of the patients were not required, since the present study did not involve a direct access by the study investigators on data of individual patients.

Data Availability Statement: The data were provided from the Federal Statistical Office of Germany (Statistisches Bundesamt, DEStatis) (source: RDC of the Federal Statistical Office and the Statistical Offices of the federal states, DRG Statistics 2005-2017, and own calculations).

Acknowledgments: We thank the Federal Statistical Office of Germany (Statistisches Bundesamt, DEStatis) for providing the pooled data for the study cohort of ischemic stroke patients in Germany 2005-2017 and the kind permission to publish these data/results.

Conflicts of Interest: K.K., J.W., V.H.S., M.A.O., I.S., T.M. and C.E.-K. report no conflict of interest. T.M. is PI of the DZHK (German Center for Cardiovascular Research), Partner Site Rhine-Main, Mainz, Germany. L.H. reports having received lecture honoraria from MSD.

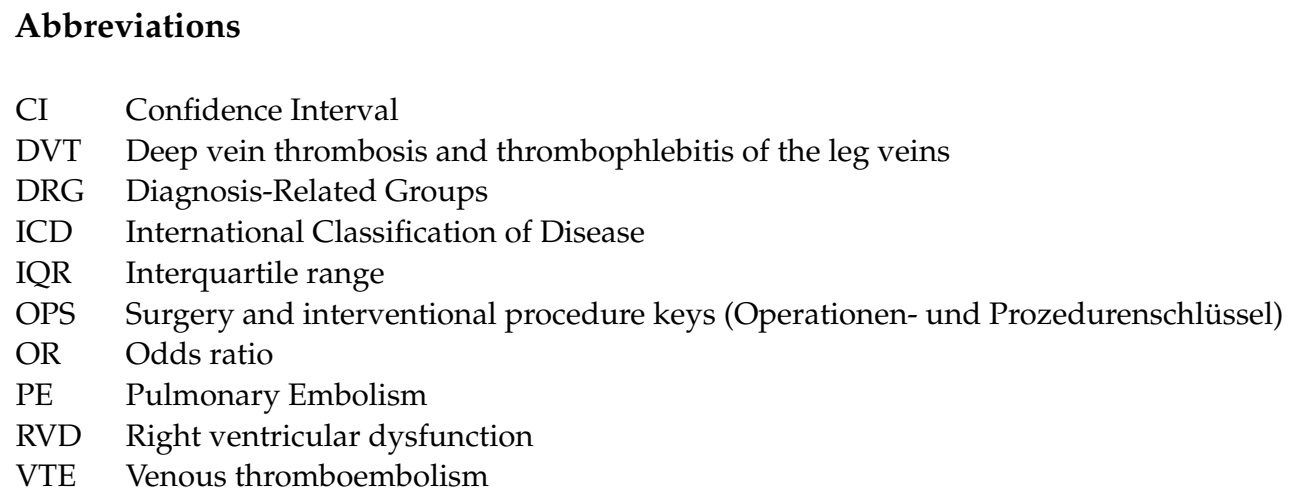

\section{References}

1. Kelly, J.; Rudd, A.; Lewis, R.; Hunt, B.J. Venous thromboembolism after acute stroke. Stroke 2001, 32, 262-267. [CrossRef]

2. Warlow, C.; Ogston, D.; Douglas, A.S. Deep venous thrombosis of the legs after strokes: Part 2-Natural history. Br. Med. J. 1976, 1, 1181-1183. [CrossRef]

3. Kelly, J.; Rudd, T.; Lewis, R.R.; Hunt, B.J. Mortality from pulmonary embolism after acute stroke: Can we do better? Age Ageing 2002, 31, 159-161. [CrossRef]

4. Wijdicks, E.F.; Scott, J.P. Pulmonary embolism associated with acute stroke. Mayo Clin. Proc. 1997, 72, 297-300. [CrossRef]

5. Sherman, D.G.; Albers, G.W.; Bladin, C.; Fieschi, C.; Gabbai, A.A.; Kase, C.S.; O'Riordan, W.; Pineo, G.F.; Investigators, P. The efficacy and safety of enoxaparin versus unfractionated heparin for the prevention of venous thromboembolism after acute ischaemic stroke (PREVAIL Study): An open-label randomised comparison. Lancet 2007, 369, 1347-1355. [CrossRef] 
6. Warlow, C.; Ogston, D.; Douglas, A.S. Deep venous thrombosis of the legs after strokes. Part I-incidence and predisposing factors. Br. Med. J. 1976, 1, 1178-1181. [CrossRef]

7. Keller, K.; Hobohm, L.; Munzel, T.; Lankeit, M.; Ostad, M.A. Impact of pulmonary embolism on in-hospital mortality of patients with ischemic stroke. J. Neurol. Sci. 2020, 419, 117174. [CrossRef]

8. Anderson, F.A., Jr.; Spencer, F.A. Risk factors for venous thromboembolism. Circulation 2003, 107, I9-I16. [CrossRef]

9. Konstantinides, S.V.; Meyer, G. The 2019 ESC Guidelines on the Diagnosis and Management of Acute Pulmonary Embolism. Eur. Heart J. 2019, 40, 3453-3455. [CrossRef]

10. Keller, K.; Hobohm, L.; Barco, S.; Schmidtmann, I.; Munzel, T.; Engelhardt, M.; Goldhofer, M.; Konstantinides, S.V.; Drees, P. Venous thromboembolism in patients hospitalized for hip joint replacement surgery. Thromb. Res. 2020, 190, 1-7. [CrossRef]

11. Keller, K.; Hobohm, L.; Barco, S.; Schmidtmann, I.; Munzel, T.; Engelhardt, M.; Eckhard, L.; Konstantinides, S.V.; Drees, P. Venous thromboembolism in patients hospitalized for knee joint replacement surgery. Sci. Rep. 2020, 10, 22440. [CrossRef]

12. Torbicki, A.; Perrier, A.; Konstantinides, S.; Agnelli, G.; Galie, N.; Pruszczyk, P.; Bengel, F.; Brady, A.J.; Ferreira, D.; Janssens, U.; et al. Guidelines on the diagnosis and management of acute pulmonary embolism: The Task Force for the Diagnosis and Management of Acute Pulmonary Embolism of the European Society of Cardiology (ESC). Eur. Heart J. 2008, $29,2276-2315$.

13. Konstantinides, S.V.; Torbicki, A.; Agnelli, G.; Danchin, N.; Fitzmaurice, D.; Galie, N.; Gibbs, J.S.; Huisman, M.V.; Humbert, M.; Kucher, N.; et al. 2014 ESC guidelines on the diagnosis and management of acute pulmonary embolism. Eur. Heart J. 2014, 35, 3033-3080. [CrossRef]

14. Keller, K.; Hobohm, L.; Munzel, T.; Ostad, M.A. Impact of concomitant deep or superficial venous thrombosis of the legs on survival of patients with pulmonary embolism. Int. J. Cardiol. 2020, 315, 92-98. [CrossRef]

15. Schwartz, T.; Hingorani, A.; Ascher, E.; Marks, N.; Shiferson, A.; Jung, D.; Jimenez, R.; Jacob, T. Pulmonary embolism without deep venous thrombosis. Ann. Vasc. Surg. 2012, 26, 973-976. [CrossRef]

16. Sorensen, H.T.; Horvath-Puho, E.; Lash, T.L.; Christiansen, C.F.; Pesavento, R.; Pedersen, L.; Baron, J.A.; Prandoni, P. Heart disease may be a risk factor for pulmonary embolism without peripheral deep venous thrombosis. Circulation 2011, 124, 1435-1441. [CrossRef]

17. Keller, K.; Prochaska, J.H.; Coldewey, M.; Gobel, S.; Ullmann, A.; Junger, C.; Lamparter, H.; Ariza, L.; Bickel, C.; Lauterbach, M.; et al. History of deep vein thrombosis is a discriminator for concomitant atrial fibrillation in pulmonary embolism. Thromb. Res. 2015, 136, 899-906. [CrossRef]

18. Ungprasert, P.; Sanguankeo, A.; Upala, S.; Suksaranjit, P. Psoriasis and risk of venous thromboembolism: A systematic review and meta-analysis. QJM 2014, 107, 793-797. [CrossRef]

19. Dumic, I.; Martin, S.; Salfiti, N.; Watson, R.; Alempijevic, T. Deep Venous Thrombosis and Bilateral Pulmonary Embolism Revealing Silent Celiac Disease: Case Report and Review of the Literature. Case Rep. Gastrointest. Med. 2017, 2017, 5236918. [CrossRef]

20. Keller, K.; Hobohm, L.; Ostad, M.A.; Karbach, S.; Espinola-Klein, C.; Munzel, T.; Gelfand, J.M.; Konstantinides, S.V.; Steinbrink, K.; Gori, T. Psoriasis and its impact on the clinical outcome of patients with pulmonary embolism. Int. J. Cardiol. 2021, 343, 114-121. [CrossRef]

21. Chung, W.S.; Lin, C.L. Increased risks of venous thromboembolism in patients with psoriasis. A Nationwide Cohort Study. Thromb. Haemost. 2017, 117, 1637-1643.

22. Ogdie, A.; Kay McGill, N.; Shin, D.B.; Takeshita, J.; Jon Love, T.; Noe, M.H.; Chiesa Fuxench, Z.C.; Choi, H.K.; Mehta, N.N.; Gelfand, J.M. Risk of venous thromboembolism in patients with psoriatic arthritis, psoriasis and rheumatoid arthritis: A general population-based cohort study. Eur. Heart J. 2018, 39, 3608-3614. [CrossRef]

23. Lee, J.J.; Pope, J.E. A meta-analysis of the risk of venous thromboembolism in inflammatory rheumatic diseases. Arthritis Res. 2014, 16, 435. [CrossRef]

24. Zoller, B.; Li, X.; Sundquist, J.; Sundquist, K. Risk of pulmonary embolism in patients with autoimmune disorders: A nationwide follow-up study from Sweden. Lancet 2012, 379, 244-249. [CrossRef]

25. Johannesdottir, S.A.; Schmidt, M.; Horvath-Puho, E.; Sorensen, H.T. Autoimmune skin and connective tissue diseases and risk of venous thromboembolism: A population-based case-control study. J. Thromb. Haemost. 2012, 10, 815-821. [CrossRef]

26. Yuhara, H.; Steinmaus, C.; Corley, D.; Koike, J.; Igarashi, M.; Suzuki, T.; Mine, T. Meta-analysis: The risk of venous thromboembolism in patients with inflammatory bowel disease. Aliment. Pharm. 2013, 37, 953-962. [CrossRef]

27. Grainge, M.J.; West, J.; Card, T.R. Venous thromboembolism during active disease and remission in inflammatory bowel disease: A cohort study. Lancet 2010, 375, 657-663. [CrossRef]

28. Reinohl, J.; Kaier, K.; Reinecke, H.; Schmoor, C.; Frankenstein, L.; Vach, W.; Cribier, A.; Beyersdorf, F.; Bode, C.; Zehender, M. Effect of Availability of Transcatheter Aortic-Valve Replacement on Clinical Practice. N. Engl. J. Med. 2015, 373, $2438-2447$. [CrossRef]

29. Keller, K.; Munzel, T.; Hobohm, L.; Ostad, M.A. Predictive value of the Kuijer score for bleeding and other adverse in-hospital events in patients with venous thromboembolism. Int. J. Cardiol. 2021, 329, 179-184. [CrossRef]

30. Keller, K.; Geyer, M.; Munzel, T.; Ostad, M.A.; Gori, T. Impact of atrial fibrillation on in-hospital mortality of ischemic stroke patients and identification of promoting factors of atrial thrombi-Results from the German nationwide inpatient sample and a single-center retrospective cohort. Medicine 2019, 98, e14086. [CrossRef] 
31. InEK GmbH-Institut für das Entgeltsystem im Krankenhaus. Deutsche Kodierrichtlinien 2018 Druckversion A4 (PDF). 2018. Available online: https://www.g-drg.de/inek_site_de/layout/set/standard/Media/Files/G-DRG-System/G-DRG-System_ 2018/Deutsche_Kodierrichtlinien_2018_Druckversion_A4_PDF (accessed on 5 June 2020).

32. Keller, K.; Hobohm, L.; Ebner, M.; Kresoja, K.P.; Munzel, T.; Konstantinides, S.V.; Lankeit, M. Trends in thrombolytic treatment and outcomes of acute pulmonary embolism in Germany. Eur. Heart J. 2020, 41, 522-529. [CrossRef]

33. Keller, K.; Hobohm, L.; Munzel, T.; Ostad, M.A. Impact of symptomatic atherosclerosis in patients with pulmonary embolism. Int. J. Cardiol. 2019, 278, 225-231. [CrossRef]

34. Armour, B.S.; Courtney-Long, E.A.; Fox, M.H.; Fredine, H.; Cahill, A. Prevalence and Causes of Paralysis-United States, 2013. Am. J. Public Health 2016, 106, 1855-1857. [CrossRef]

35. Feigin, V.L.; Norrving, B.; Mensah, G.A. Global Burden of Stroke. Circ. Res. 2017, 120, 439-448. [CrossRef]

36. Weidner, N.; Muller, O.J.; Hach-Wunderle, V.; Schwerdtfeger, K.; Krauspe, R.; Pauschert, R.; Waydhas, C.; Baumberger, M.; Goggelmann, C.; Wittgruber, G.; et al. Prevention of thromboembolism in spinal cord injury-S1 guideline. Neurol. Res. Pract. 2020, 2, 43. [CrossRef]

37. Agarwal, N.K.; Mathur, N. Deep vein thrombosis in acute spinal cord injury. Spinal Cord. 2009, 47, 769-772. [CrossRef]

38. Prandoni, P. Venous and arterial thrombosis: Two aspects of the same disease? Eur. J. Intern. Med. 2009, 20, 660-661. [CrossRef]

39. Prandoni, P.; Bilora, F.; Marchiori, A.; Bernardi, E.; Petrobelli, F.; Lensing, A.W.; Prins, M.H.; Girolami, A. An association between atherosclerosis and venous thrombosis. N. Engl. J. Med. 2003, 348, 1435-1441. [CrossRef]

40. Sorensen, H.T.; Horvath-Puho, E.; Pedersen, L.; Baron, J.A.; Prandoni, P. Venous thromboembolism and subsequent hospitalisation due to acute arterial cardiovascular events: A 20-year cohort study. Lancet 2007, 370, 1773-1779. [CrossRef]

41. Libertiny, G.; Hands, L. Deep venous thrombosis in peripheral vascular disease. Br. J. Surg. 1999, 86, 907-910. [CrossRef]

42. Keller, K.; Hobohm, L.; Munzel, T.; Lankeit, M.; Konstantinides, S.; Ostad, M.A. Impact of Systemic Atherosclerosis on Clinical Characteristics and Short-term Outcomes in Patients with Deep Venous Thrombosis or Thrombophlebitis. Am. J. Med. Sci. 2021. Online ahead of print. [CrossRef]

43. Rafi, S.; van Doormaal, F.F.; van Lienden, K.P.; Kamphuisen, P.W.; Gerdes, V.E. Venous thrombo-embolism and aortic calcifications; more evidence on the link between venous and arterial thrombosis. Thromb. Res. 2009, 124, 381-382. [CrossRef]

44. Hong, C.; Zhu, F.; Du, D.; Pilgram, T.K.; Sicard, G.A.; Bae, K.T. Coronary artery calcification and risk factors for atherosclerosis in patients with venous thromboembolism. Atherosclerosis 2005, 183, 169-174. [CrossRef]

45. Prandoni, P. Links between arterial and venous disease. J. Intern. Med. 2007, 262, 341-350. [CrossRef]

46. Clements, R.; Churilov, L.; Wahab, A.L.; Ng, L.C. Exploratory analysis of factors associated with venous thromboembolism in Victorian acute traumatic spinal cord-injured patients 2010-2013. Spinal Cord 2017, 55, 74-78. [CrossRef]

47. Alvarez Sabin, J. In-hospital mortality in stroke patients. Rev. Esp. Cardiol. 2008, 61, 1007-1009.

48. Heuschmann, P.U.; Kolominsky-Rabas, P.L.; Misselwitz, B.; Hermanek, P.; Leffmann, C.; Janzen, R.W.; Rother, J.; Buecker-Nott, H.J.; Berger, K.; German Stroke Registers Study, G. Predictors of in-hospital mortality and attributable risks of death after ischemic stroke: The German Stroke Registers Study Group. Arch. Intern. Med. 2004, 164, 1761-1768. [CrossRef]

49. Koennecke, H.C.; Belz, W.; Berfelde, D.; Endres, M.; Fitzek, S.; Hamilton, F.; Kreitsch, P.; Mackert, B.M.; Nabavi, D.G.; Nolte, C.H.; et al. Factors influencing in-hospital mortality and morbidity in patients treated on a stroke unit. Neurology 2011, 77, 965-972. [CrossRef]

50. Chamberlain, J.D.; Meier, S.; Mader, L.; von Groote, P.M.; Brinkhof, M.W. Mortality and longevity after a spinal cord injury: Systematic review and meta-analysis. Neuroepidemiology 2015, 44, 182-198. [CrossRef]

51. Yeo, J.D.; Walsh, J.; Rutkowski, S.; Soden, R.; Craven, M.; Middleton, J. Mortality following spinal cord injury. Spinal Cord 1998, 36, 329-336. [CrossRef]

52. White, R.H. The epidemiology of venous thromboembolism. Circulation 2003, 107, I4-I8. [CrossRef]

53. Dean, S.M.; Abraham, W. Venous thromboembolic disease in congestive heart failure. Congest. Heart Fail. 2010, 16, 164-169. [CrossRef]

54. Goldhaber, S.Z. Venous Thromboembolism in Heart Failure Patients: Pathophysiology, Predictability, Prevention. J. Am. Coll. Cardiol. 2020, 75, 159-162. [CrossRef]

55. Khorana, A.A. Venous thromboembolism and prognosis in cancer. Thromb. Res. 2010, 125, 490-493. [CrossRef]

56. Goto, S.; Haas, S.; Ageno, W.; Goldhaber, S.Z.; Turpie, A.G.G.; Weitz, J.I.; Angchaisuksiri, P.; Nielsen, J.D.; Kayani, G.; Farjat, A.; et al. Assessment of Outcomes among Patients With Venous Thromboembolism with and without Chronic Kidney Disease. JAMA Netw. Open 2020, 3, e2022886. [CrossRef]

57. Chhabra, H.S.; Sharawat, R.; Vishwakarma, G. In-hospital mortality in people with complete acute traumatic spinal cord injury at a tertiary care center in India-a retrospective analysis. Spinal Cord 2021. Online ahead of print. [CrossRef]

58. Claxton, A.R.; Wong, D.T.; Chung, F.; Fehlings, M.G. Predictors of hospital mortality and mechanical ventilation in patients with cervical spinal cord injury. Can. J. Anaesth. 1998, 45, 144-149. [CrossRef]

59. Ovbiagele, B. Nationwide trends in in-hospital mortality among patients with stroke. Stroke 2010, 41, 1748-1754. [CrossRef] 